\title{
ANÁLISE DA OCORRÊNCIA DE ERVA-DE-PASSARINHO NA ARBORIZAÇÃO DA UNIVERSIDADE FEDERAL DE SERGIPE, CAMPUS SÃO CRISTÓVÃO
}

\author{
Benjamin Leonardo Alves White ${ }^{1}$, Adauto de Souza Ribeiro ${ }^{2}$, Larissa Alves Secundo White ${ }^{3}$, \\ José Elvino do Nascimento Júnior ${ }^{4}$ \\ ${ }^{1}$ Biólogo, Doutorando em Desenvolvimento e Meio Ambiente, UFS, Aracaju, SE, Brasil; benjmk@ hotmail.com \\ ${ }^{2}$ Biólogo, Dr., Depto. de Biologia, UFS, Aracaju, SE, Brasil - adautosr@ufs.br \\ ${ }^{3}$ Eng ${ }^{\mathrm{a}}$ Florestal, UFS, Aracaju, SE, Brasil - larissawhite@ hotmail.com \\ ${ }^{4}$ Biólogo, UFS, São Cristóvão, SE, Brasil - jenascimentojr@gmail.com \\ Recebido para publicação: 05/06/2009 - Aceito para publicação: 22/07/2010
}

\begin{abstract}
Resumo
Este trabalho teve como objetivo analisar a ocorrência de erva-de-passarinho na arborização do campus da Universidade Federal de Sergipe, município de São Cristóvão. Para tal, todas as árvores foram identificadas, assim como as hemiparasitas. Fatores como a altura das árvores com ocorrência de hemiparasitas e o grau de infestação foram verificados. Constatou-se a presença de erva-depassarinho em $8,15 \%$ das árvores. A infestação ocorreu em quatro plantas exóticas - Azadirachta indica A. Juss., Casuarina equisetifolia Linn., Pithecellobium dulce Roxb. e Terminalia catappa Linn. - e em apenas uma planta nativa - Anacardium occidentale Linn. As espécies hemiparasitas encontradas foram Struthanthus vulgaris Mart. e Struthanthus polyrizus Mart. Verificou-se alta especificidade entre o hospedeiro Anacardium occidentale e o hemiparasita Struthanthus polyrizus. As árvores mais altas apresentaram uma maior ocorrência de hemiparasitas, e o estágio inicial de infestação, a partir da metodologia adotada, foi o de maior ocorrência.

Palavras-chave: Hemiparasita; vegetação urbana; Loranthaceae.
\end{abstract}

\begin{abstract}
Analysis of the incidence of mistletoes in the Sergipe Federal University, São Cristóvão Campus. This study has the purpose to analyze the mistletoes incidence on the trees of the Sergipe Federal University, São Cristóvão Campus. For such, all the trees with or without mistletoe had been identified as well as their respective hemiparasites. Also, factors as the height of the trees with hemiparasites, and the level of infestation were verified. The presence of mistletoes was verified in $8,15 \%$ of the trees. The infestation occurred in four exotic plants - Azadirachta indica A. Juss., Casuarina equisetifolia Linn., Pithecellobium dulce Roxb. and Terminalia catappa Linn., and in only one native plant - Anacardium occidentale Linn.. The hemiparasites found were: Struthanthus vulgaris Mart. and Struthanthus polyrizus Mart.. It was found high specificity between the host Anacardium occidentale and the hemiparasite Struthanthus polyrizus. The taller trees presented a larger occurrence of hemiparasites, and the initial stage of infestation, thought the adopted methodology, was the most common.

Keywords: Hemiparasite; urban vegetation; Loranthaceae.
\end{abstract}

\section{INTRODUÇÃO}

As ervas-de-passarinho formam um interessante grupo de plantas encontradas em todo o mundo. Elas são tidas muitas vezes como pragas, porque crescem sobre espécies agrícolas e afetam a qualidade da arborização urbana. Entretanto essas plantas são muito importantes em ecossistemas naturais como fonte de alimentos para as aves (CAZETTA; GALETTI, 2003).

Existem muitas controvérsias com relação à sua classificação taxonômica. Segundo Cazetta; Galetti (2003), existem cerca de 700 espécies, que se dividem em 24 gêneros pertencentes a quatro famílias (Eremolepidaceae, Loranthaceae, Misodendraceae e Viscaceae). Já segundo Watson; Dallwitz 
(1992), as ervas-de-passarinho são classificadas em apenas uma família (Loranthaceae), com aproximadamente 75 gêneros e 1000 espécies. O sistema de classificação APGII reconhece a família Loranthaceae com 70 gêneros e 800 espécies possuindo distribuição pantropical, sendo que no Brasil ocorrem 10 gêneros e aproximadamente 100 espécies (SOUZA; LORENZI, 2005).

Essas plantas são popularmente conhecidas como "erva-de-passarinho" devido à estreita dependência das aves como dispersoras de suas sementes. Tais aves apresentam alterações no tubo digestivo - ingerem a polpa dos frutos e regurgitam ou defecam a semente, deixando-a cair na casca das árvores. Em geral, as sementes são defecadas nos galhos, mas algumas vezes podem cair no solo, onde não se desenvolvem (CAZETTA; GALETTI, 2003).

Quando a semente adere no ramo da planta hospedeira, ela emite uma raiz modificada, conhecida como haustório, que se insere no xilema do hospedeiro, de onde retira água e sais minerais para seu processo de fotossíntese. São consideradas hemiparasitas, pois retiram apenas a seiva bruta do hospedeiro, diferentemente das plantas parasitas, que sugam a seiva elaborada e, consequentemente, não realizam a fotossíntese (TATTAR, 1978; ROTTA, 2001).

Essas plantas se fixam nos galhos e troncos da planta hospedeira, onde se desenvolvem e ocupam partes, ou, em alguns casos, a totalidade da copa, reduzindo a eficiência fotossintética da hospedeira. Árvores com alto grau de infestação por erva-de-passarinho são mais predispostas ao ataque de insetos e mais susceptíveis a estresses ambientais do que indivíduos saudáveis da mesma espécie. Elas acabam tendo sua taxa de crescimento reduzida, o que pode levá-las a um estado de declínio. Em determinados casos, a sua ocorrência pode ser um fator relevante a ser monitorado, pois, em desequilíbrio, compromete a arquitetura das árvores e, por se proliferar com facilidade, pode comprometer toda uma plantação agrícola ou um programa de arborização urbana (TATTAR, 1978; NORTON; CARPENTER, 1998; CAZETTA; GALLETTI, 2003; ARRUDA, 2004).

Algumas espécies de hemiparasitas são denominadas "especialistas", por serem encontradas em uma pequena quantidade de hospedeiros. Outras são denominadas "generalistas", devido ao fato de estarem presentes em uma grande diversidade de espécies hospedeiras (NORTON; REID, 1997).

Em comunidades heterogêneas, pode ser mais vantajoso às hemiparasitas serem generalistas, o que permite crescer com sucesso em todos os hospedeiros em potencial (NORTON; REID, 1997). A especificidade da hemiparasita por determinado hospedeiro pode ser favorecida em situações em que os hospedeiros são relativamente abundantes, como em monoculturas, por exemplo. Além disso, Norton; Carpenter (1998) citam que hemiparasitas especializados possuem maior eficiência na captura de recursos do hospedeiro.

Dantas (2005) observou que tanto a altura quanto a idade são variáveis que influenciam positivamente o processo parasito-hospedeiro. Segundo o mesmo autor, estudando a ocorrência da ervade-passarinho Psittacanthus dichrous Mart. na Kielmeyera rugosa Choisy, as árvores mais altas foram mais susceptíveis à infestação, por serem mais comumente visitadas por pássaros dispersores. Já os indivíduos mais velhos, que são em sua maioria os mais altos, estão há mais tempo expostos à infestação, e essa soma de fatores favorece a ocorrência de hemiparasitas.

No Brasil, pouco se conhece a respeito da sua interação com os hospedeiros, já que os estudos envolvendo Loranthaceae foram predominantemente de anatomia e embriologia. Isso pode ser reflexo de uma pequena quantidade de pesquisas de coletas botânicas para Loranthaceae, bem como dados relativos aos hospedeiros (ARRUDA, 2004).

Este estudo teve como objetivo analisar a infestação das ervas-de-passarinho nas árvores da Universidade Federal de Sergipe, Campus de São Cristóvão, com a finalidade de identificar as espécies hospedeiras e hemiparasitas, quantificar o grau de infestação segundo a metodologia de Oliveira; Kappel (1994) e verificar se as árvores mais altas são mais susceptíveis ao parasitismo. O trabalho se justifica na alta ocorrência de árvores em decadência ou mortas devido à incidência de ervas-de-passarinho na área de estudo e na necessidade da compreensão das relações ecológicas entre as espécies de hemiparasitas e as plantas parasitadas.

\section{MATERIAS E MÉTODOS}

O local de estudo está situado nas dependências do Campus da Universidade Federal de Sergipe, município de São Cristóvão, nas coordenadas $10^{\circ} 55^{\prime} \mathrm{S}$ e $37^{\circ} 06^{\prime} \mathrm{L}$, compreendendo uma área 
aproximadamente de 60 hectares. O clima, segundo a classificação de Köppen, é Aw (clima tropical com estação seca de inverno). Seu terreno é baixio de planície, característico de cidades litorâneas, e a vegetação encontra-se descaracterizada da vegetação original de Mata Atlântica, sendo encontradas diversas espécies exóticas, que foram plantadas com objetivo ornamental.

Os dados foram levantados de abril a setembro de 2007. Como método de amostragem, foram demarcadas cinco parcelas de $550 \mathrm{~m}$ de comprimento por $100 \mathrm{~m}$ de largura, distando $100 \mathrm{~m}$ entre si, amostrando uma área total de 27,5 hectares, aproximadamente $50 \%$ da área total do Campus. Todas as árvores e as hemiparasitas encontradas dentro das parcelas foram contabilizadas e identificadas no herbário da Universidade Federal de Sergipe. As árvores não hemiparasitadas foram contabilizadas como um todo, enquanto que as árvores com presença de hemiparasitas foram contabilizadas de acordo com a espécie.

As espécies infestadas tiveram sua altura medida para posterior comparação com a altura de árvores aleatórias não parasitadas, através do teste "t Student" (ZAR, 1999). Como grau de inclusão, apenas árvores não hemiparasitadas maiores que 2 metros foram medidas, devido à não ocorrência de erva-de-passarinho, no local de estudo, em árvores menores que esta altura.

Nas espécies arbóreas que apresentaram hemiparasitismo, foi empregado o método de Oliveira; Kappel (1994) para verificar o grau de infestação, assim descritos: Baixo, quando a incidência de hemiparasitas era inferior a $1 / 4$ da copa do hospedeiro; Média, quando a partir de $1 / 4$ e inferior a $1 / 2$; e Alta, quando igual a $1 / 2$ ou mais. Segundo Leal et al. (2006), o método descrito por Oliveira; Kappel (1994) demonstra ser o mais adequado, por considerar intervalo de grau de infestação mais próximo que outros métodos existentes.

\section{RESULTADOS E DISCUSSÃO}

\section{Vegetação}

As espécies arbóreas encontradas nas parcelas estudadas estão listadas na tabela 1.

Após a conclusão do levantamento, foi constatada a existência de 903 espécimes arbóreas dentro das parcelas amostradas no campus da Universidade Federal de Sergipe, distribuídas em 40 espécies, 38 gêneros e 16 famílias, sendo Fabaceae e Anacardiaceae as famílias com o maior número de espécies. Algumas espécies encontradas na área de estudo são comuns nas ruas da cidade de Aracaju, Sergipe. Segundo Matos et al. (2007), as árvores da família Fabaceae, Bignoniaceae e Moraceae são as mais comuns na arborização de ruas do centro de Aracaju, com destaque para a espécie Pithecellobium dulce. Já Resende et al. (2009), também no município de Aracaju, constataram uma maior ocorrência das espécies arbóreas Pithecellobium dulce, Terminalia catappa e Licania tomentosa, todas elas abundantes dentro do campus da Universidade Federal de Sergipe.

A frequente utilização de espécies exóticas na arborização do campus São Cristóvão da Universidade Federal de Sergipe e do município de Aracaju é um fator preocupante, pois descaracteriza o ambiente natural e reduz a riqueza e diversidade da fauna não adaptada às espécies não nativas.

\section{Índice de infestação entre hospedeiros e hemiparasitas}

Dentre 903 árvores encontradas dentro das parcelas estudadas, 73 estavam hemiparasitadas por erva-de-passarinho $(8,15 \%)$. Esses dados se assemelham com trabalhos realizados em arborização urbana, como o de Girnos et al. (1994), que encontraram incidência de 8,62\% de erva-de-passarinho na arborização de ruas de Porto Alegre (RS), e de Ruschel; Leite (2002), na arborização urbana no centro da cidade de Lajeado (RS), com 9,40\%. Entretanto existem estudos, em outras localidades, com índices maiores de infestação, como para Leal et al. (2006), que descreveram presença de hemiparasitas em 28,19\% das árvores utilizadas na arborização de ruas de Curitiba, e Arruda (2004), que relatou ocorrência de erva-de-passarinho em 17,7\% das árvores em uma área de Cerrado em Minas Gerais. Essa diferença no índice de infestação pode ser devido à existência ou não de práticas de controle, ao tipo de vegetação existente e às condições climáticas locais.

De um total de 40 espécies arbóreas encontradas no local de estudo, apenas nove delas (Adenanthera pavonina, Anadenanthera macrocarpa, Anacardium occidentale, Azadirachta indica, Casuarina equisetifolia, Mimosa caesalpiniifolia, Pithecellobium dulce, Terminalia catappa e Thespesia populnea) sofreram hemiparasitismo (Tabela 2). 
Tabela 1. Lista das espécies arbóreas encontradas no Campus da Universidade Federal de Sergipe.

Table 1. List of trees species found in the Sergipe Federal University.

\begin{tabular}{|c|c|c|c|}
\hline Família & Nome científico & Nome comum & Origem \\
\hline \multirow{5}{*}{ Anacardiaceae } & Schinus terebinthifolius Raddi & Aroeira-vermelha & Nativa \\
\hline & Myracrodruon urundeuva Fr. All. & Aroeira-do-sertão & Nativa \\
\hline & Anacardium occidentale Linn. & Caju & Nativa \\
\hline & Tapirira guianensis Aubl. & Pau-pombo & Nativa \\
\hline & Mangifera indica Linn. & Manga & Exótica \\
\hline \multirow[t]{2}{*}{ Apocynaceae } & Hancornia speciosa Gómez & Mangaba & Nativa \\
\hline & Stenolobium stans Linn. & Ipê-mirim & Exótica \\
\hline Arecaceae & Cocos nucifera Linn. & Coqueiro & Nativa \\
\hline \multirow[t]{2}{*}{ Bignoniaceae } & Crescentia cujete Linn. & Cueiro & Exótica \\
\hline & Tabebuia caraiba Mart. & Craibeira & Nativa \\
\hline Bombacaceae & Pachira aquatica Aubl. & Castanha-do-maranhão & Nativa \\
\hline Casuarinaceae & Casuarina equisetifolia Linn. & Casuarina & Exótica \\
\hline Chrysobalanaceae & Licania tomentosa Benth. & Oiti & Nativa \\
\hline Combretaceae & Terminalia catappa Linn. & Castanheira & Exótica \\
\hline \multirow[t]{15}{*}{ Fabaceae } & Senna multijuga Rich. & Canafístula & Nativa \\
\hline & Delonix regia Hook. & Flamboyant & Exótica \\
\hline & Caesalpinia echinata Lam. & Pau-brasil & Nativa \\
\hline & Caesalpinia ferrea Mart. & Pau-ferro & Nativa \\
\hline & Mimosa hostilis Benth. & Jurema-vermelha & Exótica \\
\hline & Enterolobium contortisiliquum Vell. & Tamboril, timbaúva & Nativa \\
\hline & Mimosa caesalpiniifolia Benth. & Sabiá & Nativa \\
\hline & Leucaena leucocephala Lam. & Leucena & Exótica \\
\hline & Prosopis juliflora $\mathrm{Sw}$. & Algaroba & Exótica \\
\hline & Adenanthera pavonina Linn. & Tento-carolina & Exótica \\
\hline & Anadenanthera macrocarpa Benth. & Angico-de-caroço & Nativa \\
\hline & Pithecellobium dulce Roxb. & Mata-fome & Exótica \\
\hline & Clitoria fairchidiana $\mathrm{R}$. Howard & Sombreiro & Nativa \\
\hline & Andira fraxinifolia Benth. & Angelim & Nativa \\
\hline & Erythrina velutina Willd. & Mulungu & Nativa \\
\hline Malpighiaceae & Byrsonima sericea DC. & Murici & Nativa \\
\hline Malvaceae & Thespesia populnea Benth. & Cebiá & Exótica \\
\hline \multirow[t]{3}{*}{ Moraceae } & Artocarpus integrifolia Linn. & Jaca & Exótica \\
\hline & Ficus retusa Linn. & Figo & Exótica \\
\hline & Ficus elastica Roxb. & Figo & Exótica \\
\hline Meliaceae & Azadirachta indica A. Juss. & Neen & Exótica \\
\hline \multirow[t]{3}{*}{ Myrtaceae } & Syzygium jambolanum Lam. & Jamelão & Exótica \\
\hline & Psidium guajava Linn. & Goiaba & Nativa \\
\hline & Corymbia citriodora Hook. & Eucalipto & Exótica \\
\hline Salicaceae & Casearia silvestris Swartz & Camarão & Nativa \\
\hline Sapindaceae & Sapindus saponaria Linn. & Saboneteira & Nativa \\
\hline
\end{tabular}

As duas espécies de erva-de-passarinho encontradas pertencem à família Loranthaceae: Struthanthus polyrhizus Mart. e Struthanthus vulgaris Mart. Reif; Andreata (2006) citam a ocorrência dessas espécies no estado do Rio de Janeiro. Rotta (2001) faz referência a essas duas espécies como estando dentre as mais comuns nas árvores das ruas de Curitiba.

A Casuarina equisetifolia apresentou o maior índice de ocorrência de erva-de-passarinho, aparentando ser uma espécie bastante susceptível a hemiparasitas. Segundo Caires (2003), essa mesma espécie sofreu infestação por Phthirusa ovata Pohl (Loranthaceae) na arborização urbana de Brasília, DF. Outro trabalho, realizado na Austrália, catalogou a presença de Decaisnina triflora Span. (Loranthaceae) na Casuarina equisetifolia, além de Lysiana subfalcata Hook. (Loranthaceae) em Azadirachta indica e Amyema conspicua Bail. (Loranthaceae) em Thespesia populnea (DOWNEY, 1998). 
Tabela 2. Hospedeiros e hemiparasitas encontrados.

Table 2. Hosts and hemiparasites found.

\begin{tabular}{|c|c|c|c|c|}
\hline \multirow[b]{2}{*}{ Árvore hospedeira } & \multicolumn{2}{|c|}{ Quantidade de árvores } & \multirow{2}{*}{$\begin{array}{c}\text { Percentagem de } \\
\text { árvores } \\
\text { hemiparasitadas }\end{array}$} & \multirow[b]{2}{*}{ Hemiparasita } \\
\hline & $\begin{array}{c}\text { Com } \\
\text { hemiparasitas } \\
\end{array}$ & $\begin{array}{c}\text { Sem } \\
\text { hemiparasitas }\end{array}$ & & \\
\hline Anacardium occidentale & 17 & 50 & $25,8 \%$ & $\begin{array}{l}\text { Struthanthus polyrhizus (16) } \\
\text { e Struthanthus vulgaris (1) }\end{array}$ \\
\hline Casuarina equisetifolia & 15 & 0 & $100 \%$ & Struthanthus vulgaris \\
\hline Terminalia catappa & 14 & 53 & $20,9 \%$ & Struthanthus vulgaris \\
\hline Pithecellobium dulce & 12 & 39 & $23,5 \%$ & Struthanthus vulgaris \\
\hline Azadirachta indica & 11 & 94 & $10,5 \%$ & Struthanthus vulgaris \\
\hline $\begin{array}{l}\text { Anadenanthera } \\
\text { macrocarpa }\end{array}$ & 1 & 1 & $50 \%$ & Struthanthus vulgaris \\
\hline Mimosa caesalpiniifolia & 1 & 1 & $50 \%$ & Struthanthus vulgaris \\
\hline Thespesia populnea & 1 & 1 & $50 \%$ & Struthanthus vulgaris \\
\hline Adenanthera pavonina & 1 & 1 & $50 \%$ & Struthanthus vulgaris \\
\hline
\end{tabular}

Os resultados demonstram claramente que existem alguns aspectos característicos na relação entre parasita/hospedeiro. Certas espécies arbóreas, por exemplo, podem ter atributos que as tornem mais susceptíveis do que outras, apresentando, consequentemente, um alto índice de infestação. Leal et al. (2006) citam que a infestação está diretamente relacionada à espécie do hospedeiro, já que algumas se apresentam bastante sensíveis, enquanto outras podem ser tolerantes ou até mesmo resistentes.

S. polyrhizus apresentou alta especificidade, infectando apenas as árvores de Anacardiun occidentale. Já $S$. vulgaris apresentou comportamento generalista, sendo encontrada em diferentes espécies de hospedeiro. Apenas no hospedeiro Anacardium occidentale ocorreu hemiparasitismo pelas duas espécies de erva-de-passarinho ao mesmo tempo.

Observou-se, na área de estudo, que 98,63\% dos hemiparasitas apresentavam-se isolados, ou seja, apenas uma espécie hemiparasita por hospedeiro. Leal et al. (2006) citam a ocorrência de uma pequena quantidade de hemiparasitas de duas distintas espécies ocorrendo em um mesmo hospedeiro na cidade de Curitiba. A pequena existência de diferentes espécies de hemiparasitas em um mesmo hospedeiro pode significar que talvez existam mecanismos que promovam a sua ocorrência em estado isolado.

\section{Grau de infestação}

Segundo a metodologia de Oliveira; Kappel (1994), aproximadamente $78 \%$ das árvores hemiparasitadas enquadravam-se na classe "Baixo" de infestação. Essa classe diferiu estatisticamente das classes "Médio" e "Alto", com graus de infestação de 18\% e 4\%, respectivamente.

Leal et al. (2006), em estudo realizado em árvores urbanas de Curitiba, encontraram o grau de infestação no estágio inicial mais frequente $(68,84 \%)$, seguido pelo estágio mediano $(13,49 \%)$ e estágio final $(17,66 \%)$.

Devido ao baixo grau de infestação por erva-de-passarinho presente na maioria das árvores amostradas, há a possibilidade de controle desses hemiparasitas sem maior comprometimento das árvores e através de metodologias de baixo custo, como, por exemplo, a poda ou a retirada manual. Árvores com graus de infestação "Médio" e "Alto" necessitam de monitoramento e cuidados especiais, para que não entrem em estado de declínio e morram.

\section{Altura das árvores hemiparasitadas}

A altura das árvores hemiparasitadas apresentou uma média de $7,92 \mathrm{~m}$, superior às das não parasitadas, que foi de $5,98 \mathrm{~m}$. Essa diferença foi significativamente grande para rejeitar a hipótese nula de que árvores hemiparasitadas apresentam uma mesma altura que as não hemiparasitadas $(t=3,33$, $\mathrm{p}<0,05, \mathrm{gl}=144)$ (Figura 1).

Segundo Dzeferos (2003), em pequenas árvores de Eucalyptus spp. da Austrália, tem-se encontrado um menor número de parasitas de Amyema preissii Miq. (Loranthaceae) do que em árvores grandes. Já Lamont (1985) mediu as alturas e diâmetros das hospedeiras e confirmou que árvores maiores 
possuem maior infestação que árvores menores. Dados similares foram encontrados para Viscaceae em Quercus spp. (THOMSON; MAHALL, 1983) e para a Loranthaceae Amyema spp. em Eucalyptus spp. (LAMONT, 1985; REID; LANGE, 1988).

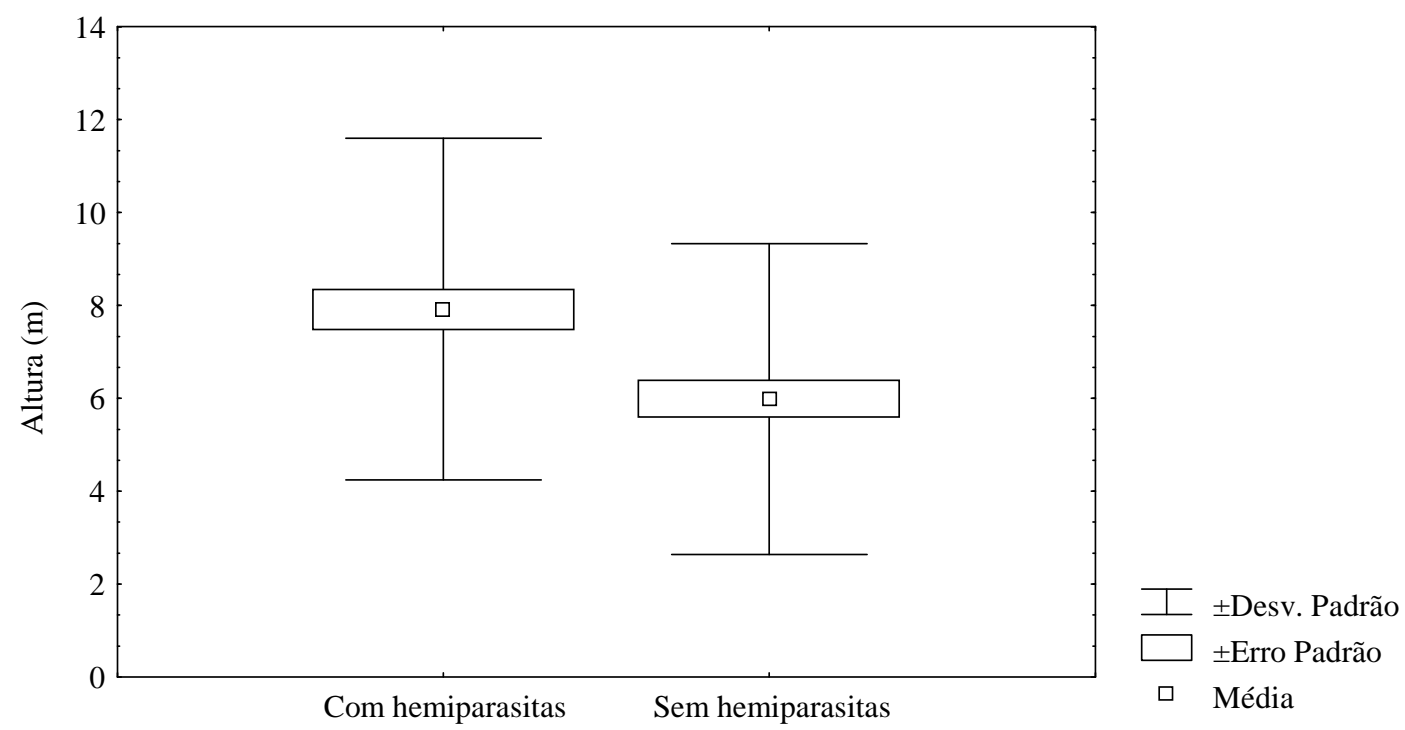

Figura 1. Média, erro padrão e desvio padrão da altura das árvores com e sem presença de hemiparasitas. Figure 1. Mean, standard error and standard deviation of the height from the trees with and without hemiparasites.

A maior incidência de hemiparasitas em árvores de grande porte é explicada por trabalhos como o de Reid et al. (1999), que afirma que grandes árvores são mais susceptíveis a reinfestação, pois suas sementes caem novamente na planta hóspede e há, assim, a germinação de uma nova planta parasita. Arruda (2004) afirma que a maior ocorrência de hemiparasitas em árvores mais altas pode ocorrer em razão do modo de utilização das árvores pelas aves dispersoras. Estudos do comportamento das aves dispersoras das sementes de ervas-de-passarinho, como o de Monteiro et al. (1992), que denotam a preferência da ave Tersina viridis (Illiger, 1811) por poleiros em galhos mais altos e com copas mais densas, corroboram a teoria de que as ervas-de-passarinho costumam ocorrer em árvores mais altas.

\section{CONCLUSÕES}

- $8,15 \%$ das 903 árvores dentro das parcelas estudadas apresentaram incidência de infestação por ervade-passarinho.

- Dentre as 40 espécies arbóreas encontradas, apenas nove apresentaram infestação por erva-depassarinho.

- A espécie nativa Anacardium occidentale e as exóticas Azadirachta indica, Casuarina equisetifolia, Pithecellobium dulce e Terminalia catappa foram as que apresentaram maior quantidade de espécimes infestados.

- A hemiparasita Struthanthus polyrizus apresentou comportamento especialista, parasitando apenas Anacardium occidentale, enquanto que Struthanthus vulgaris apresentou comportamento generalista.

- $78 \%$ das árvores hemiparasitadas enquadraram-se na classe "Baixo" de infestação, $18 \%$ na classe "Médio" e 4\% na classe "Alto".

- As árvores hemiparasitadas apresentaram uma altura significativamente maior do que a altura das não hemiparasitadas. 


\section{REFERÊNCIAS}

ARRUDA, R. S. Especificidade de hospedeiros por Struthanthus aff. polyanthus (Loranthaceae) em uma área de cerrado, Uberlândia, Minas Gerais. 2004. 34 f. Dissertação (Mestrado em Ecologia e Conservação de Recursos Naturais) - Universidade Federal de Uberlândia, Uberlândia, 2004.

CAIRES, C. S.; SOUZA, R. G.; ZANATTA, G. V.; GOMES, B. M. Levantamento preliminar dos hospedeiros de Phthirusa ovata (Pohl) Eichl. (Loranthaceae) na arborização urbana de Brasília, DF. In: Congresso Nacional de Botânica, 54., 2003, Belém. Anais... Belém: Sociedade Botânica do Brasil, 2003.

CAZETTA, E.; GALETTI, M. Ecologia das ervas-de-passarinho. Ciência Hoje, v. 33, n. 194, p. 72-74, 2003.

DANTAS, T. V. D. Relação entre a estrutura populacional de Kielmeyera Rugosa (Clusiaceae) e o índice de infestação de Psittacanthus Dichrous Mart. ex. Schult (Loranthaceae) no habitat de Areias Brancas, na Serra de Itabaiana, Sergipe. 2005. 35 f. Monografia (Graduação em Biologia) Universidade Federal de Sergipe, São Cristóvão, 2005.

DOWNEY, P. O. An inventory of host species for each aerial mistletoe species (Loranthaceae and Viscaceae) in Australia. Cunninghamia, v. 5, n. 3, p. 685-720, 1998.

GIRNOS, E. C.; CAMPOS, D.; COSTA, C. L.; MACHADO, M. R. B.; MARTINS, F. C.; MARTINS, L. M.; PEREIRA, C. Q. B.; REIS, J. C. M.; SANTOS, R. C. Sobre a ocorrência de "erva-de-passarinho" nas praças de São Luís (MA). In: Congresso Brasileiro de Arborização Urbana, 3., 1994, São Luís. Anais... São Luís: SBAU, 1994. p. 327-334.

LAMONT B. Host distribution, potassium content, water relation and control af two co-occurring mistletoe species. Journal of the Royal Society of Western Australia, v. 68, p. 21-25, 1985.

LEAL, L.; BUJOKAS, W. M.; BIONDI, D. Análise da infestação de erva-de-passarinho na arborização de ruas de Curitiba, PR. Revista Floresta, Curitiba, v. 36, n. 3, p. 323-330, 2006.

MATOS, E. C. A.; NASCIMENTO-JÚNIOR, J. E.; MARIANO, D. L. S.; OLIVEIRA, A. L. Arborização do bairro centro da cidade de Aracaju, SE. In: Congresso de Ecologia do Brasil, 8, 2007, Caxambu. Anais... Caxambu: Sociedade Brasileira de Ecologia, 2007. 2 p.

MONTEIRO, R. F.; MARTINS, R. P.; YAKAMOTO, K. Host specificity and seed dispersal of Psittacanthus robustus (Loranthaceae) in south-east Brazil. Journal of Tropical Ecology, v. 8, p. 307314, 1992.

NORTON, D. A.; CARPENTER, M. A. Mistletoes as parasites: host specificity and speciation. Trend in Ecology and Evolution, v. 13, p. 101-105, 1998.

NORTON, D. A.; REID, N. Lessons in ecosystem management from management of threatened and pest loranthaceous mistletoes in New Zealand and Australia. Conservation Biology, v. 11, p. 759-769, 1997.

OLIVEIRA, F. B.; KAPPEL, R. B. Incidência de erva-de-passarinho na arborização de ruas de Porto Alegre. In: Congresso Brasileiro de Arborização Urbana, 3., 1994, São Luís. Anais... São Luís: SBAU, 1994. p. 335-346.

REID, N.; LANGE, R. T. Host specificity, dispersion and persistence through drought of two arid zone mistletoe. Australian Journal Botany, v. 36, p. 299-313, 1988.

REID, N.; LAVOREL, S.; SMITH, M. S. Spred of mistletoes (Amyema preissii) in fragmented Australian Woodlands: a simulation study. Landscape Ecology, v. 14, p. 147-160, 1999.

REIF, C.; ANDREATA, R. H. P. Sinopse de "ervas-de-passarinho" do estado do Rio de Janeiro, Brasil. Pesquisa Botânica, São Leopoldo, n. 57, p. 255-274, 2006. 
RESENDE, W. X.; SOUZA, H. T. R.; MELO e SOUZA, R. Índices de áreas verdes públicas: uma avaliação fitogeográfica da qualidade ambiental em Aracaju. In: Simpósio Brasileiro de Geografia Física Aplicada, 13., 2009, Viçosa. Anais... Viçosa: Universidade Federal de Viçosa, 2009. 20 p.

RUSCHEL, D.; LEITE, S. L. C. Arborização urbana em uma área da cidade de Lajeado, Rio Grande do Sul, Brasil. Caderno de Pesquisa Série Biologia, Santa Cruz do Sul, v. 14, n. 1, p. 7-24, 2002.

SOUZA, V. C.; LORENZI, H. Botânica Sistemática: Guia ilustrado para identificação das famílias Angiospermas da flora brasileira, baseado na APGII. Nova Odessa: Instituto Plantarum de Estudos da Flora Ltda., 2005. 640 p.

TATTAR, T. A. Diseases of shade trees. New York: Academic, 1978. 361 p.

THOMSON, V. E.; MAHALL, B. E. Host specificity by a mistletoe, Phoradendron villosum on three oak species in California. Botanical Gazette, v. 144, p. 124-131, 1983.

WATSON, L.; DALLWITZ, M. J. The families of flowering plants: descriptions, illustrations, identification, and information retrieval. 1992. Disponível em: <http://delta-intkey.com>. Acesso em: 31 de maio de 2009.

ZAR, J. H. Biostatistical analysis. 4. ed. New Jersey: Prentice Hall, 1999. 663 p. 